International Journal of Pure and Applied Mathematics

Volume 86 No. 3 2013, 501-514

ISSN: 1311-8080 (printed version); ISSN: 1314-3395 (on-line version)

url: http://www.ijpam.eu

doi: http://dx.doi.org/10.12732/ijpam.v86i3.4

ijpam.eu

\title{
COMBINATORIAL RESULTS FOR SEMIGROUPS OF ORDER-PRESERVING FULL TRANSFORMATIONS WITH PATTERN OF RANGE
}

\author{
Phichet Jitjankarn \\ Division of Mathematics \\ School of Science \\ Walailak University \\ Nakhon Si Thammarat, 80161, THAILAND
}

\begin{abstract}
Let $\mathcal{O}[n]$ and $\mathcal{C}[n]$ be the semigroup of all order-preserving and all order-preserving and regressive transformations on the set $[n]=\{1, \ldots, n\}$, respectively. In this paper, we give a pattern on $[n]$. Combinatorial properties on subsemigroups of $\mathcal{O}[n]$ with restricted range to the pattern are investigated.
\end{abstract}

AMS Subject Classification: 20M20, 11B39

Key Words: order-preserving transformation semigroups, regressive transformation semigroups, Fibonacci numbers, Catalan numbers

\section{Introduction}

For a set $[n]=\{1, \ldots, n\} . \mathcal{T}[n]$ denotes the full transformation semigroup on $[n]$. We shall call a transformation $\alpha:[n] \rightarrow[n]$ order-preserving if $x \leq y$ implies $x \alpha \leq y \alpha$ for all $x, y \in[n]$, and regressive (or order-decreasing) if $x \alpha \leq x$ for all $x \in[n]$. We denote by $\mathcal{O}[n]$ the semigroup of all full order-preserving transformations on $[n]$ and denote by $\mathcal{R}[n]$ the semigroup of all full regressive transformations on $[n]$. Let $\mathcal{C}[n]=\mathcal{O}[n] \cap \mathcal{R}[n]$.

Received: March 1, 2013

(c) 2013 Academic Publications, Ltd. url: www.acadpubl.eu 
On these semigroups, combinatorial results are well understood by now. For example, Howie in [4] showed that $|\mathcal{O}[n]|=\left(\begin{array}{c}2 n-1 \\ n-1\end{array}\right)$ and $|E \mathcal{O}[n]|=F_{2 n}$, where $E \mathcal{O}[n]$ is the set of all idempotents of $\mathcal{O}[n]$ and $F_{2 n}$ is the alternate Fibonacci number given by $F_{1}=F_{2}=1$. Higgins in [2] and later in (alternative version) $[6,7]$ Laradji and Umar proved that $|\mathcal{C}[n]|=\frac{1}{n+1}\left(\begin{array}{c}2 n \\ n\end{array}\right)$, the $n$th Catalan number $C_{n}$.

In 1975, Symons [11] introduced the subsemigroup of full transformation with restricted range and later Sanwong in [10] studied its regularity. Recently in $[5,12]$ some classifications on subsemigroups of full order-preserving (regressive) transformations with restricted range were investigated. These papers motivated the study of combinatorial properties of semigroups of transformations with restricted range.

For $t \in \mathbb{N}$ and $n_{1}, \ldots, n_{t}, m_{1}, \ldots, m_{t} \in \mathbb{N}_{0}$, a pattern $\left[m_{1 n_{1}} m_{2 n_{2}} \cdots m_{t n_{t}}\right]$ is defined to be

$$
\left\{1,2, \ldots, \sum_{i=1}^{t}\left(m_{i}+n_{i}\right)\right\}
$$

having the structure of two disjoint subsets

$$
\left\{1, \ldots, m_{1}\right\} \cup \bigcup_{k=1}^{t}\left(\sum_{i=1}^{k}\left(m_{i}+n_{i}\right)+\left\{1, \ldots, m_{k+1}\right\}\right),
$$

denoted by $\left\lceil m_{1 n_{1}} m_{2 n_{2}} \cdots m_{t n_{t}}\right\rceil$, and

$$
\left(m_{1}+\left\{1, \ldots, n_{1}\right\}\right) \cup \bigcup_{k=1}^{t-1}\left(m_{1}+\sum_{i=1}^{k}\left(n_{i}+m_{i+1}\right)+\left\{1, \ldots, n_{k+1}\right\}\right),
$$

denoted by $\left\lfloor m_{1 n_{1}} m_{2 n_{2}} \cdots m_{t n_{t}}\right\rfloor$, respectively.

For example, $\left[1_{2} 3_{1}\right]=\{1,2,3,4,5,6,7\}$ with $\left\lceil 1_{2} 3_{1}\right\rceil=\{1,4,5,6\}$ and $\left\lfloor 1_{2} 3_{1}\right\rfloor$ $=\{2,3,7\}$.

Note that for $i=1, \ldots, t$, if $m_{i}=0$, then

$$
\left[m_{1 n_{1}} \cdots m_{i n_{i}} \cdots m_{t n_{t}}\right]=\left[m_{1 n_{1}} \cdots m_{i-1 n_{i-1}+n_{i}} \cdots m_{t n_{t}}\right]
$$

and if $n_{i}=0$, then

$$
\left[m_{1 n_{1}} \cdots m_{i n_{i}} \cdots m_{t n_{t}}\right]=\left[m_{1 n_{1}} \cdots\left(m_{i}+m_{i+1}\right)_{n_{i+1}} \cdots m_{t n_{t}}\right] .
$$

For brevity, we shall omit to write 0 in any positions of the pattern if it is 0 . For example, $[25]:=\left[0_{2} 5_{0}\right],[2]:=\left[0_{2}\right]$ and $[5]:=\left[5_{0}\right]$. When all positions are 0 , we will write $[0]$ instead.

Let $\mathcal{S}$ be the set containing all these elements. We define a binary operation on $\mathcal{S}$ in natural way of the concatenation, for example, $\left[1_{2} 1\right]\left[1_{3} 2_{1}\right]=\left[1_{2} 2_{3} 2_{1}\right]$, 
$[3]\left[1_{1} 3\right]=\left[4_{1} 3\right]$ and $\left[{ }_{1}\right]\left[{ }_{1} 3_{3}\right]=\left[{ }_{2} 3_{3}\right]$. Then $\mathcal{S}$ is a free monoid with $[0]$ as the identity element.

For $[\mathbf{x}] \in \mathcal{S}$, by considering $\lceil\mathbf{x}\rceil(\lfloor\mathbf{x}\rfloor)$ as the skeleton of $[\mathbf{x}]$ and grouping elements in $\lfloor\mathbf{x}\rfloor(\lceil\mathbf{x}\rceil)$ into classes, we have that each class contains all elements in $\lfloor\mathbf{x}\rfloor(\lceil\mathbf{x}\rceil)$ which has no elements in $\lceil\mathbf{x}\rceil(\lfloor\mathbf{x}\rfloor)$ lies between them and it is said to be a lower(upper)-class of $[\mathbf{x}]$. A lower-class containing $a$ and an upper-class containing $b$ of $[\mathbf{x}]$ are denoted by $\underline{a}$ and $\bar{b}$, respectively.

Let $\mathcal{T}[\mathbf{x}]$ be the full transformation semigroup under composition of all mapping of $[\mathbf{x}]$ into $\lceil\mathbf{x}\rceil$. For $\mathcal{O}[\mathbf{x}], \mathcal{R}[\mathbf{x}]$ and $\mathcal{C}[\mathbf{x}]$ are defined analogously.

We already know that the number of integral solutions of the equation

$$
x_{1}+x_{2}+\cdots+x_{n+1}=n-1
$$

that satisfy $x_{i} \geq 0$ is equal to the cardinality of $\mathcal{O}[n],\left(\begin{array}{c}2 n-1 \\ n-1\end{array}\right)$. For example, when $n=7$

- $\left(x_{1}, x_{2}, x_{3}, x_{4}, x_{5}, x_{6}, x_{7}, x_{8}\right)=(1,0,0,1,1,3,0,0)$ is the representation of

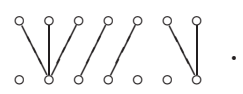

- $\left(x_{1}, x_{2}, x_{3}, x_{4}, x_{5}, x_{6}, x_{7}, x_{8}\right)=(0,0,1,1,0,2,1,1)$ is the representation of

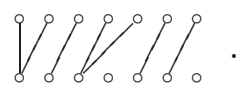

It is clear that the cardinality of the set containing all order-preserving from $\{1, \ldots, p\}$ to $\{1, \ldots, q\}$ is $\left(\begin{array}{c}p+q-1 \\ p\end{array}\right)$. Consequently, the following result is obtained.

Theorem 1.1. The cardinality of $\mathcal{O}\left[m_{1 k_{1}} m_{2 k_{2}} \cdots k_{t-1} m_{t}\right]$ is $\left(\begin{array}{c}2 m+k-1 \\ m-1\end{array}\right)$ where $m=m_{1}+\cdots+m_{t}$ and $k=k_{1}+\cdots+k_{t-1}$.

The paper is organized as follows: In Section 2 and 3, we deal with cardinalities of $\mathcal{E O}\left[m_{1 k_{1}} m_{2 k_{2}} \cdots k_{t-1} m_{t}\right], \mathcal{C}\left[m_{k}\right]$ and $\mathcal{C}\left[m_{k} n\right]$. In Section 4, cardinalities of some equivalence classes are investigated.

\section{The Number of Idempotents}

To find the cardinality of $E \mathcal{O}\left[m_{1 k_{1}} m_{2 k_{2}} \cdots_{k_{t-1}} m_{t}\right]$, our strategy will be to look at the sets having some types as follows: 
For $\alpha \in E \mathcal{O}\left[m_{1 k_{1}} m_{2 k_{2}} \cdots k_{t-1} m_{t}\right]$, if there are exactly distinct $n$ lowerclasses, $\underline{g_{1}}, \ldots, \underline{g_{n}}$ of $\left[m_{1 k_{1}} m_{2 k_{2}} \cdots k_{t-1} m_{t}\right]$ such that for $i \in\{1, \ldots, n\}$,

$$
\left|\left(\underline{g_{i}} \cup\left\{\min \underline{g_{i}}-1, \max \underline{g_{i}}+1\right\}\right) \alpha\right|=1,
$$

then $\alpha$ is called a type of $n$ closed lower-classes (or $t-1-n$ open lower-classes).

For $s \in \mathbb{N}_{0}$, let $U_{s}\left[m_{1 k_{1}} m_{2 k_{2}} \cdots_{k_{t-1}} m_{t}\right]$ and $V_{s}\left[m_{1 k_{1}} m_{2 k_{2}} \cdots_{k_{t-1}} m_{t}\right]$ stand for the set of all idempotents in $\mathcal{O}\left[m_{1 k_{1}} m_{2 k_{2}} \cdots_{k_{t-1}} m_{t}\right]$ of the type $s$ open lower-classes and $s$ closed lower-classes, respectively.

Example. In $E \mathcal{O}\left[2_{2} 2_{2} 1\right]$,

$$
\begin{aligned}
& \alpha=\left(\begin{array}{lllllllll}
1 & 2 & 3 & 4 & 5 & 6 & 7 & 8 & 9 \\
1 & 2 & 2 & 5 & 5 & 5 & 5 & 5 & 5
\end{array}\right) \in V_{1}\left[2_{2} 2_{2} 1\right]=U_{1}\left[2_{2} 2_{2} 1\right] \\
& \beta=\left(\begin{array}{lllllllll}
1 & 2 & 3 & 4 & 5 & 6 & 7 & 8 & 9 \\
1 & 2 & 2 & 2 & 2 & 9 & 9 & 9 & 9
\end{array}\right) \in V_{2}\left[2_{2} 2_{2} 1\right]=U_{0}\left[22_{2} 2_{2} 1\right] .
\end{aligned}
$$

Note that in the structure of $\alpha$, there is the shape of a right triangle, $\nabla$, from $(\{5,6\} \cup \underline{7} \cup\{9\}) \alpha=\{5\}$,

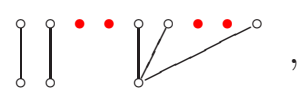

whereas in the structure of $\beta$, there is the shape of two right triangles, $\nabla \nabla$, from $(\{2\} \cup \underline{3} \cup\{5\}) \beta=\{2\}$ and $(\{6\} \cup \underline{7} \cup\{9\}) \beta=\{9\}$,

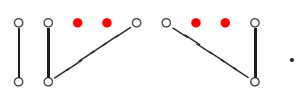

We denote

$$
V_{1}\left[m_{1 k_{1}} \cdots_{k_{i-1}} m_{i \triangleright} \cdots_{k_{t-1}} m_{t}\right] \text { and } V_{1}\left[m_{1 k_{1}} \cdots_{k_{i-1}} m_{i \triangleleft} \cdots_{k_{t-1}} m_{t}\right]
$$

as two subsets of $V_{1}\left[m_{1 k_{1}} \cdots_{k_{i-1}} m_{i k_{i}} \cdots_{k_{t-1}} m_{t}\right]$, with the closed lower-class at $\underline{g_{i}}=\left(m_{i}+\sum_{j=1}^{i-1}\left(m_{j}+k_{j}\right)\right)+\left\{1, \ldots, k_{i}\right\}$ in the structure of $\nabla$ and $\nabla$, respectively. For example,

$$
V_{1}\left[2_{2} 2_{2} 1\right]=V_{1}\left[2_{\triangleleft} 2_{2} 1\right] \dot{\cup} V_{1}\left[2_{\triangleright} 2_{2} 1\right] \dot{\cup} V_{1}\left[2_{2} 2_{\triangleleft} 1\right] \dot{\cup} V_{1}\left[2_{2} 2_{\triangleright} 1\right] \text {. }
$$

For $V_{n}\left[m_{1 k_{1}} m_{2 k_{2}} \cdots k_{t-1} m_{t}\right]$, it can be written as a disjoint union of $2^{n}\left(\begin{array}{c}t-1 \\ n\end{array}\right)$ sets defined in analogous way. For example,

$$
V_{2}\left[2_{2} 2_{2} 1\right]=V_{2}\left[2_{\triangleright} 2_{\triangleleft} 1\right] \dot{\cup} V_{2}\left[2_{\triangleright} 2_{\triangleright} 1\right] \dot{\cup} V_{2}\left[2_{\triangleleft} 2_{\triangleleft} 1\right] \dot{U} V_{2}\left[2_{\triangleleft} 2_{\triangleright} 1\right] .
$$

The following lemma is obtained immediately. 
Lemma 2.1. $\left|E \mathcal{O}\left[m_{1 k_{1}} m_{2 k_{2}} \cdots_{k_{t-1}} m_{t}\right]\right|=\sum_{i=0}^{t-1}\left|V_{i}\left[m_{1 k_{1}} m_{2 k_{2}} \cdots_{k_{t-1}} m_{t}\right]\right|$.

Lemma 2.2. For $V_{1}\left[m_{k} n\right]$, we have that

$$
\left|V_{1}\left[m_{\triangleleft} n\right]\right|=F_{2 m-1} F_{2 n} \text { and }\left|V_{1}\left[m_{\triangleright} n\right]\right|=F_{2 m} F_{2 n-1} .
$$

Proof. To illustrate the cardinality of $V_{1}\left[m_{\triangleleft} n\right]$, we consider the following cases:

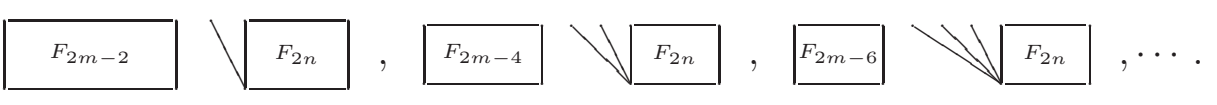

Then $\left|V_{1}\left[m_{\triangleleft} n\right]\right|=\left(\sum_{i=1}^{m} F_{2(m-i)}+1\right) F_{2 n}$. By using the Fibonacci identity: $\sum_{i=1}^{n} F_{2 i}=F_{2 n+1}-1$, it follows that $\left|V_{1}\left[m_{\triangleleft} n\right]\right|=F_{2 m-1} F_{2 n}$ as wanted.

For the rest, it can be proved in the same fashion.

From Lemma 2.1 and 2.2, the following proposition is obtained.

Proposition 2.3. The cardinality of $E \mathcal{O}\left[m_{k} n\right]$ is

$$
(k+1) F_{2 m} F_{2 n}+F_{2 m-1} F_{2 n}+F_{2 m} F_{2 n-1} .
$$

As a consequence of Proposition 2.3 and Howie's result, by taking $k=0$, we have the following conclusion.

Corollary 2.4. The cardinality of $E \mathcal{O}\left[m_{k} n\right]$ is $k F_{2 m} F_{2 n}+F_{2(m+n)}$.

\begin{tabular}{ccccccc}
\hline & 1 & 2 & 3 & 4 & 5 & 6 \\
\hline 1 & $k+3$ & $3 k+8$ & $8 k+21$ & $21 k+55$ & $55 k+144$ & $144 k+377$ \\
2 & $3 k+8$ & $9 k+21$ & $24 k+55$ & $63 k+144$ & $165 k+377$ & $432 k+987$ \\
3 & $8 k+21$ & $24 k+55$ & $64 k+144$ & $168 k+377$ & $440 k+987$ & $1152 k+2584$ \\
4 & $21 k+55$ & $63 k+144$ & $168 k+377$ & $441 k+987$ & $1155 k+2584$ & $3024 k+6765$ \\
5 & $55 k+144$ & $165 k+377$ & $440 k+987$ & $1155 k+2584$ & $3025 k+6765$ & $7920 k+17711$ \\
6 & $144 k+377$ & $432 k+987$ & $1152 k+2584$ & $3024 k+6765$ & $7920 k+17711$ & $20736 k+46368$ \\
\hline
\end{tabular}

Table 1: The cardinality of $E \mathcal{O}\left[m_{k} n\right]$

Proposition 2.5. The cardinality of $E \mathcal{O}\left[m_{1 k_{1}} m_{2 k_{2}} m_{3}\right]$ is $k_{1} k_{2} F_{2 m_{1}} F_{2 m_{2}} F_{2 m_{3}}+k_{1} F_{2 m_{1}} F_{2\left(m_{2}+m_{3}\right)}+k_{2} F_{2\left(m_{1}+m_{2}\right)} F_{2 m_{3}}+F_{2\left(m_{1}+m_{2}+m_{3}\right)}$. 
Proof. It is clear that $\left|V_{0}\left[m_{1 k_{1}} m_{2 k_{2}} m_{3}\right]\right|=\left(k_{1}+1\right)\left(k_{2}+1\right) F_{2 m_{1}} F_{2 m_{2}} F_{2 m_{3}}$. Since $V_{1}\left[m_{1 k_{1}} m_{2 k_{2}} m_{3}\right]$ is

$$
V_{1}\left[m_{1 \triangleright} m_{2 k_{2}} m_{3}\right] \dot{\cup} V_{1}\left[m_{1 \triangleleft} m_{2 k_{2}} m_{3}\right] \dot{\cup} V_{1}\left[m_{1 k_{1}} m_{2 \triangleright} m_{3}\right] \dot{\cup} V_{1}\left[m_{1 k_{1}} m_{2 \triangleleft} m_{3}\right]
$$

and $V_{2}\left[m_{1 k_{1}} m_{2 k_{2}} m_{3}\right]$ is

$$
V_{2}\left[m_{1 \triangleright} m_{2 \triangleright} m_{3}\right] \dot{\cup} V_{2}\left[m_{1 \triangleleft} m_{2 \triangleleft} m_{3}\right] \dot{\cup} V_{2}\left[m_{1 \triangleleft} m_{2 \triangleright} m_{3}\right] \dot{\cup} V_{2}\left[m_{1 \triangleright} m_{2 \triangleleft} m_{3}\right],
$$

by applying Lemma 2.2, we have

$$
\begin{aligned}
\left|V_{1}\left[m_{1 k_{1}} m_{2 k_{2}} m_{3}\right]\right|= & \left(k_{1}+1\right)\left[F_{2 m_{1}} F_{2 m_{2}} F_{2 m_{3}-1}+F_{2 m_{1}} F_{2 m_{2}-1} F_{2 m_{3}}\right] \\
& +\left(k_{2}+1\right)\left[F_{2 m_{1}} F_{2 m_{2}-1} F_{2 m_{3}}+F_{2 m_{1}-1} F_{2 m_{2}} F_{2 m_{3}}\right], \\
\left|V_{2}\left[m_{1 k_{1}} m_{2 k_{2}} m_{3}\right]\right|= & F_{2 m_{1}} F_{2 m_{2}-1} F_{2 m_{3}-1}+F_{2 m_{1}-1} F_{2 m_{2}-1} F_{2 m_{3}} \\
& +F_{2 m_{1}-1} F_{2 m_{2}} F_{2 m_{3}-1}+F_{2 m_{1}} F_{2 m_{2}-2} F_{2 m_{3}} .
\end{aligned}
$$

Using the Fibonacci identity: $F_{m+n}=F_{m-1} F_{n}+F_{m} F_{n+1}$ and Howie's result when $k_{1}=k_{2}=0$, our proof is finished.

From Proposition 2.5, we have an identity of 3-term Fibonacci numbers (see also in [3] and [9]): $F_{2\left(m_{1}+m_{2}+m_{3}\right)}$ is

$2 F_{2 m_{1}} F_{2 m_{2}} F_{2 m_{3}}+F_{2 m_{1}} F_{2 m_{2}+1} F_{2 m_{3}-1}+F_{2 m_{1}+1} F_{2 m_{2}-1} F_{2 m_{3}}+F_{2 m_{1}-1} F_{2 m_{2}} F_{2 m_{3}+1}$.

As a polynomial in the variables $k_{1}, \ldots, k_{t-1}$ with coefficient in $\mathbb{Z}$, the leading coefficient of $k_{i_{1}} \cdots k_{i_{d}}$ in $\left|E \mathcal{O}\left[m_{1 k_{1}} m_{2 k_{2}} \cdots k_{t-1} m_{t}\right]\right|$ is denoted by $c\left(k_{i_{1}} \cdots k_{i_{d}}\right)$.

Lemma 2.6. In $\left|E \mathcal{O}\left[m_{1 k_{1}} m_{2 k_{2}} \cdots k_{t-1} m_{t}\right]\right|, c\left(k_{i} k_{i+n_{1}} \cdots k_{i+n_{1}+\cdots+n_{r}}\right)$ is

$$
F_{2\left(m_{1}+\cdots+m_{i}\right)} F_{2\left(m_{i+1}+\cdots+m_{i+n_{1}}\right)} \cdots F_{2\left(m_{i+n_{1}+\cdots+n_{r}+1}+\cdots+m_{t}\right)} \cdot
$$

Proof. By Corollary 2.4 and Proposition 2.5, the result holds for $\left|E \mathcal{O}\left[m_{1 k_{1}} m_{2}\right]\right|$ and $\left|E \mathcal{O}\left[m_{1 k_{1}} m_{2 k_{2}} m_{3}\right]\right|$. We prove the result by induction on the number of lower-classes. Suppose the result holds for $\left|E \mathcal{O}\left[m_{1 k_{1}} m_{2 k_{2}} \cdots_{k_{p-1}} m_{p}\right]\right|$ where $p=2, \ldots, t-1$.

To find $c\left(k_{i} k_{i+n_{1}} \cdots k_{i+n_{1}+\cdots+n_{r}}\right)$, let $q \in\left\{i, i+n_{1}, \ldots, i+n_{1}+\cdots+n_{r}\right\}$. By considering all terms having $k_{q}$ as a factor in $\left|E \mathcal{O}\left[m_{1 k_{1}} m_{2 k_{2}} \cdots_{k_{t-1}} m_{t}\right]\right|$, it suffices to consider the set of all idempotent such that $\underline{g_{q}}=\left(m_{i}+\sum_{j=1}^{q-1}\left(m_{j}+\right.\right.$ $\left.\left.k_{j}\right)\right)+\left\{1, \ldots, k_{q}\right\}$ is an open lower-class, namely $A_{q}$. Then

$$
\left|A_{q}\right|=\left(k_{q}+1\right)\left|E \mathcal{O}\left[m_{1 k_{1}} \cdots_{k_{q-1}} m_{q}\right]\right| \cdot\left|E \mathcal{O}\left[m_{q+1 k_{q+1}} \cdots_{k_{t-1}} m_{t}\right]\right| .
$$


WLOG, we let $q=i+\left(n_{1}+\cdots+n_{d}\right)$. Then

$$
c\left(k_{i} k_{i+n_{1}} \cdots k_{i+n_{1}+\cdots+n_{d-1}} k_{i+n_{1}+\cdots+n_{d}} k_{i+n_{1}+\cdots+n_{d+1}} \cdots k_{i+n_{1}+\cdots+n_{r}}\right)
$$

is the product of

$$
c\left(k_{i} k_{i+n_{1}} \cdots k_{i+n_{1}+\cdots+n_{d-1}}\right) \text { and } c\left(k_{i+n_{1}+\cdots+n_{d+1}} \cdots k_{i+n_{1}+\cdots+n_{r}}\right)
$$

in $\left|E \mathcal{O}\left[m_{1 k_{1}} \cdots_{k_{q-1}} m_{q}\right]\right|$ and $\left|E \mathcal{O}\left[m_{q+1 k_{q+1}} \cdots_{k_{t-1}} m_{t}\right]\right|$, respectively. By induction, the proof is finished.

Theorem 2.7. The cardinality of $E \mathcal{O}\left[m_{1 k_{1}} m_{2 k_{2}} \cdots_{k_{t-1}} m_{t}\right]$ is

$$
F_{2\left(m_{1}+m_{2}+\cdots m_{t}\right)}+\sum_{A \in \mathcal{P}(\{1, \ldots, t-1\})\{\emptyset\}}\left[c\left(\prod_{i \in A} k_{i}\right) \cdot \prod_{i \in A} k_{i}\right]
$$

Proof. From Lemma 2.6 and by using Howie's result when $k_{1}=k_{2}=\cdots=$ $k_{t-1}=0$, our proof is finished.

From theorem above, the cardinality of $E \mathcal{O}\left[m_{1 k_{1}} m_{2 k_{2}} m_{3 k_{3}} m_{4}\right]$ is

$$
\begin{aligned}
& F_{2\left(m_{1}+m_{2}+m_{3}+m_{4}\right)} \\
+ & k_{1} k_{2} k_{3} F_{2 m_{1}} F_{2 m_{2}} F_{2 m_{3}} F_{2 m_{4}}+k_{1} F_{2 m_{1}} F_{2\left(m_{2}+m_{3}+m_{4}\right)}+k_{2} F_{2\left(m_{1}+m_{2}\right)} F_{2\left(m_{3}+m_{4}\right)} \\
+ & k_{3} F_{2\left(m_{1}+m_{2}+m_{3}\right)} F_{2 m_{4}}+k_{1} k_{2} F_{2 m_{1}} F_{2 m_{2}} F_{2\left(m_{3}+m_{4}\right)}+k_{1} k_{3} F_{2 m_{1}} F_{2\left(m_{2}+m_{3}\right)} F_{2 m_{4}} \\
& +k_{2} k_{3} F_{2\left(m_{1}+m_{2}\right)} F_{2 m_{3}} F_{2 m_{4}} .
\end{aligned}
$$

Corollary 2.8. The cardinality of $E \mathcal{O}\left[m_{k} m_{k} \cdots_{k} m\right]$, (with $n-1$ lowerclasses), is

$$
\begin{aligned}
& F_{2 n m}+\sum_{p_{1}+p_{2}=n} F_{2\left(p_{1} m\right)} F_{2\left(p_{2} m\right)} k+\sum_{p_{1}+p_{2}+p_{3}=n} F_{2\left(p_{1} m\right)} F_{2\left(p_{2} m\right)} F_{2\left(p_{3} m\right)} k^{2} \\
& +\cdots+\sum_{p_{1}+\cdots+p_{n-1}=n} F_{2\left(p_{1} m\right)} \cdots F_{2\left(p_{n-1} m\right)} k^{n-2}+\left(F_{2 m}\right)^{n} k^{n-1} .
\end{aligned}
$$

\section{The Number of Regressive Maps}

We aim now to find the cardinality of $\mathcal{C}\left[m_{k}\right]$ and $\mathcal{C}\left[m_{k} n\right]$, respectively. Let $l(n, t)$ be the cardinality of the set $L(n, t)$ containing all maps in $\mathcal{C}[n]$ whose $n$ is sent to $n-t$. It is clear that $l(n, 0)=l(n, 1)=C_{n-1}$. 


\begin{tabular}{lccc}
\hline & 2 & 3 & 4 \\
\hline 1 & $3+k$ & $8+6 k+k^{2}$ & $21+25 k+9 k^{2}+k^{3}$ \\
2 & $21+9 k$ & $144+126 k+27 k^{2}$ & $987+1305 k+567 k^{2}+81 k^{3}$ \\
3 & $144+64 k$ & $2584+2304 k+512 k^{2}$ & $46368+62080 k+27648 k^{2}+4096 k^{3}$ \\
4 & $987+441 k$ & $46368+41454 k+9261 k^{2}$ & $2178309+2921625 k+1305801 k^{2}+194481 k^{3}$ \\
5 & $6765+3025 k$ & $832040+744150 k+166375 k^{2}$ & $102334155+137289625 k+61392375 k^{2}+9150625 k^{3}$ \\
6 & $46368+20736 k$ & $14930352+13353984 k+2985984 k^{2}$ & $4807526976+6449932800 k+2884460544 k^{2}+429981696 k^{3}$ \\
\hline
\end{tabular}

Table 2: The cardinality of $E \mathcal{O}\left[m_{k} m_{k} \cdots_{k} m\right]$ with $n-1$ lower-classes

To find $l(n, t)$ when $t \geq 2$, let $M(n, t, k)$ be the set containing all maps $\alpha$ in $L(n, t)$ such that $(n-t-k+1) \alpha=n-t-k$ for $k \in\{0,1, \ldots, n-t-1\}$ and let

$$
S(n, t, k):=M(n, t, k) \bigcup_{i=0}^{k-1} S(n, t, i) .
$$

It is clear that $|S(n, t, 0)|=C_{n-t}$.

Remark 3.1. The following results are obtained immediately:

1. $L(n, t)=S(n, t, 0) \dot{\cup} S(n, t, 1) \dot{\cup} \cdots \dot{\cup} S(n, t, n-t-1)$.

2. $\mathcal{C}[n]=L(n, 0) \dot{\cup} L(n, 1) \dot{\cup} \cdots \dot{\cup} L(n, n-1)$.

3. $\mathcal{C}\left[n_{m}\right]=L(n+m, m) \dot{\cup} L(n+m, m+1) \dot{\cup} \ldots \dot{\cup} L(n+m, n+m-1)$.

Consider $S(n, t, k)$ for all $k=0,1, \ldots, n-t-1$. Given $\alpha \in S(n, t, k)$. We firstly consider the structure of $\alpha$ as follows:

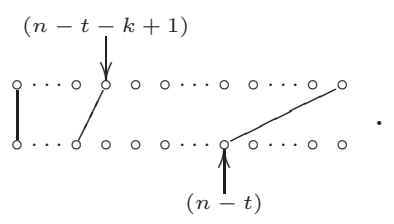

It is clear that

$$
\left|\left\{\left.\alpha\right|_{\{1, \ldots, n-t-k\}}: \alpha \in S(n, t, k)\right\}\right|=C_{n-t-k} .
$$

We denote $s(n, t, k)$ as the cardinality of $\left\{\left.\alpha\right|_{\{n-t-k+2, \ldots, n-1\}}: \alpha \in S(n, t, k)\right\}$.

Lemma 3.2. The following statements hold:

(i) $s(n, 2, k)=C_{k}$ for $k=0,1, \ldots, n-3$.

(ii) $s(n, t, k)=\left|\mathcal{C}\left[(k+1)_{t-3}\right]\right|$ for $t \geq 3$ and $k=0,1, \ldots, n-t-1$. 
Proof. (i) Let $\alpha \in S(n, 2, k)$. Suppose $k>0$. Then $(n-1) \alpha \leq n-3$. We consider $\left.\alpha\right|_{\{n-k, \ldots, n-1\}}:\{n-k, \ldots, n-1\} \rightarrow\{n-2-k, \ldots, n-3\}$ where $R=\{n-2-k, \ldots, n-3\}$,

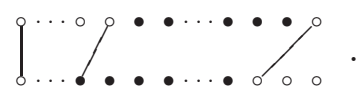

Then $\left.\alpha\right|_{D_{2}}$ acts as an order-preserving map $\beta$ on $\{1, \ldots, k\}$. It remains to show that $\beta$ is regressive. Suppose there is $x \in\{1, \ldots, k\}$ such that $(x) \beta>x$. Since $\alpha$ is regressive, it implies that either $(x) \beta=x+1$ or $(x) \beta=x+2$. Then $\alpha \in S(n, 2, t)$ for some $t<k$ which is a contradiction. Then $s(n, 2, k)=C_{k}$.

(ii) For any $\alpha \in S(n, t, k)$, we have that $\{n-t-k+2, \ldots, n-1\} \alpha \subseteq$ $\{n-t-k, \ldots, n-t\}$,

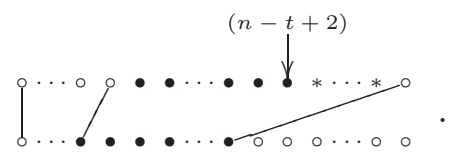

To show that $\left\{\left.\alpha\right|_{\{n-t-k+2, \ldots, n-1\}}: \alpha \in S(n, t, k)\right\}$ and the set $B$ containing all order-preserving and regressive maps from $\{1, \ldots, t+k-2\}$ to $\{1, \ldots, k+1\}$ have the same cardinality, we consider the restriction of $\alpha \in S(n, t, k)$ on $\{n-t+$ $3, \ldots, n-1\}$. It is clear that $\{n-t+3, \ldots, n-1\} \alpha \subseteq\{(n-t+2) \alpha, \ldots, n-t\}$. It remains to consider the restriction of $\alpha \in S(n, t, k)$ on $\{n-t-k+2, \ldots, n-t+2\}$. By the same argument as before, our proof is finished.

Remark 3.3. From Remark 3.1 and Lemma 3.2 , we have that

1. $s(n, 3, k)=C_{k+1}$ for $k=0,1, \ldots, n-4$.

2. For $t \geq 4$ and $k=0,1, \ldots, n-t-1$,

$$
s(n, t, k)=C_{t+k-2}-\sum_{i=0}^{t-4} l(t+k-2, i) .
$$

3. For $t \geq 2$,

$$
l(n, t)=\sum_{k=0}^{n-t-1} C_{n-t-k} s(n, t, k) .
$$

We also have an identity for Catalan numbers as follows:

Proposition 3.4. For $m \geq n+1$,

$$
C_{n-1}=\sum_{i=0}^{n-2} s(m, n-i, i)
$$


Proof. Since $\mathcal{C}[n+1]=L(n+1,0) \dot{\cup} L(n+1,1) \dot{\cup} L(n+1,2) \dot{\cup} \ldots \dot{\cup} L(n+$ $1, n)$, it follows that $C_{n+1}$ is

$$
\begin{aligned}
& 2 C_{n}+\sum_{i=2}^{n} l(n+1, i) \\
& =2 C_{n}+\sum_{i=2}^{n} \sum_{j=0}^{n-i}|S(n+1, i, j)| \\
& =2 C_{n}+\left[C_{n-1} s(n+1,2,0)+C_{n-2} s(n+1,2,1)+\cdots+C_{1} s(n+1,2, n-2)\right] \\
& \quad+\left[C_{n-2} s(n+1,3,0)+\cdots+C_{1} s(n+1,3, n-3)\right] \\
& \quad \vdots \\
& \quad+\left[C_{2} s(n+1, n-1,0)+C_{1} s(n+1, n-1,1)\right] \\
& \quad+C_{1} s(n+1, n, 0) .
\end{aligned}
$$

The proof is finished, by applying the recursion for Catalan numbers: $C_{n+1}=$ $\sum_{i=0}^{n} C_{i} C_{n-i}$.

\begin{tabular}{cccccccccc}
\hline & 0 & 1 & 2 & 3 & 4 & 5 & 6 & 7 & 8 \\
\hline 2 & 1 & 1 & 2 & 5 & 14 & 42 & 132 & 429 & 1430 \\
3 & 1 & 2 & 5 & 14 & 42 & 132 & 429 & 1430 & 4862 \\
4 & 1 & 3 & 9 & 28 & 90 & 297 & 1001 & 3432 & 11934 \\
5 & 1 & 4 & 14 & 48 & 165 & 572 & 2002 & 7072 & 25194 \\
6 & 1 & 5 & 20 & 75 & 275 & 1001 & 3640 & 13260 & 48450 \\
7 & 1 & 6 & 27 & 110 & 429 & 1638 & 6188 & 23256 & 87210 \\
\hline
\end{tabular}

Table 3: $s(n, t, k)$

Using the recursion for Catalan numbers $C_{n+1}=\sum_{i=0}^{n} C_{i} C_{n-i}$, the following results are obtained.

1. $l(n, 0)=l(n, 1)=C_{n-1}$.

2. $l(n, 2)=C_{n-1}-C_{n-2}$.

3. $l(n, 3)=C_{n-1}-2 C_{n-2}$.

From Remark 3.3, for $t \geq 4, l(n, t)$ is obtained by following recurrence relation:

$$
l(n, t)=\sum_{k=0}^{n-t-1} C_{n-t-k}\left(C_{t+k-2}-\sum_{i=0}^{t-4} l(t+k-2, i)\right) .
$$

Hence the following result is directly obtained. 
Proposition 3.5. For $k, m, n \in \mathbb{N}$, we have that:
(i) $\left|\mathcal{C}\left[m_{k}\right]\right|=C_{m+k}-\sum_{i=0}^{k-1} l(m+k, i)$.
(ii) $\left|\mathcal{C}\left[m_{k} n\right]\right|=\sum_{i=0}^{n}\left|\mathcal{C}\left[m_{k+i}\right]\right| \cdot C_{n-i}$.

\section{Cardinalities of Some Equivalence Classes}

There are several equivalence relations on semigroups, which play an important role in the structure theory. For $\alpha \in \mathcal{T}(X)$, the symbol $\pi_{\alpha}$ will denote the partition of $X$ induced by $\alpha$, namely,

$$
\pi_{\alpha}=\left\{x \alpha^{-1}: x \in \operatorname{ran} \alpha\right\} .
$$

The Green's relations on $\mathcal{T}(X)$ are described by

$$
\begin{array}{ll}
\alpha \mathcal{L} \beta & \text { if and only if } \operatorname{ran} \alpha=\operatorname{ran} \beta \\
\alpha \mathcal{R} \beta & \text { if and only if } \pi_{\alpha}=\pi_{\beta} .
\end{array}
$$

For a nonempty subset $Y$ of a chain $X$, given a transformation $\alpha: X \rightarrow Y$, the skeleton of $\alpha$ consists of the partial map of $\alpha$ by restricted its domain on $Y$ and $\operatorname{ran} \alpha$. Define an equivalence relation $\mathcal{K}$ on $\mathcal{T}(X)$ by

$$
\alpha \mathcal{K} \beta \quad \text { if and only if }\left.\quad \alpha\right|_{Y}=\left.\beta\right|_{Y} \text { and } \operatorname{ran} \alpha=\operatorname{ran} \beta \text {. }
$$

This relation helps us to classify semigroups of full regressive transformations with restricted range (see $[5,12]$ ).

We denote $\mathcal{L}$-class, $\mathcal{R}$-class and $\mathcal{K}$-class containing $\alpha$ by $L_{\alpha}, R_{\alpha}$ and $K_{\alpha}$, respectively.

Proposition 4.1. Let $\alpha$ be a right identity in $\mathcal{O}\left[m_{1 k_{1}} m_{2 k_{2}} \cdots_{k_{t-1}} m_{t}\right]$. Then $\left|L_{\alpha}\right|=\left|K_{\alpha}\right|=\left(k_{1}+1\right) \cdots\left(k_{t-1}+1\right)$ and $\left|R_{\alpha}\right|=1$.

Proof. Let $\alpha$ be a right identity in $\mathcal{O}\left[m_{1 k_{1}} m_{2 k_{2}} \cdots_{k_{t-1}} m_{t}\right]$. We have that $\operatorname{ran} \alpha=\left\lceil m_{1 k_{1}} m_{2 k_{2}} \cdots k_{t-1} m_{t}\right\rceil$. It follows that $L_{\alpha}$ and $K_{\alpha}$ are the same. To build such a map $\beta \in L_{\alpha}$, by order-preserving property, there are $|\underline{a}|+1$ ways in each lower-class $\underline{a}$ for sending via $\beta$ into its range. Then we have the result. Next, we consider $R_{\alpha}$. Since $\alpha$ is a right identity, it follows that for each $A \in \pi_{\alpha}$, $A \cap \operatorname{ran} \alpha=\left\{n_{A}\right\}$ and $A=\left(n_{A}\right) \alpha^{-1}$. As $\alpha$ is order-preserving, we have that $A$ is one of the following convex sets: $\left\{n_{A}\right\},\left\{n_{A}, n_{A}+1, \ldots, n_{A}+m\right\}$ or $\left\{n_{A}, n_{A}-1, \ldots, n_{A}-n\right\}$ for some $m, n \in \mathbb{N}$. These imply that $R_{\alpha}$ is trivial. 
It is known [8] that every regressive subsemigroup $S$ of $\mathcal{T}(X)$ when $X$ is an ordered set, is $\mathcal{R}$-trivial, that is, $\left|R_{\alpha}\right|=1$ for all $\alpha \in S$. In next propositions, we find the cardinality of $\mathcal{K}$-trivial subsets of $\mathcal{O}\left[m_{1 k_{1}} m_{2 k_{2}} \cdots_{k_{t-1}} m_{t}\right]$ and $\mathcal{C}\left[m_{1 k_{1}} m_{2 k_{2}} \cdots_{k_{t-1}} m_{t}\right]$, respectively. It is straightforward to verify the following lemma.

Lemma 4.2. For $\alpha \in \mathcal{O}\left[m_{k} n\right], K_{\alpha}$ is trivial if and only if one of the following statements hold:

(i) $|(m+\{0,1, \ldots, k, k+1\}) \alpha|=1$.

(ii) $k=1$ and $((m+1) \alpha) \alpha^{-1}=\{m+1\}$.

Theorem 4.3. Suppose $k_{i}>1$ for all $i=1, \ldots, t-1$ and $m=m_{1}+\cdots+m_{t}$. Then we have:

(i) $\mid\left\{\alpha \in \mathcal{O}\left[m_{1 k_{1}} m_{2 k_{2}} \cdots k_{t-1} m_{t}\right]: K_{\alpha}\right.$ is trivial $\} \mid$ is $\left(\begin{array}{c}2 m-t \\ m-1\end{array}\right)$.

(ii) $\mid\left\{\alpha \in \mathcal{O}\left[m_{11} m_{21} \cdots_{1} m_{t}\right]: K_{\alpha}\right.$ is trivial $\} \mid$ is

$$
\begin{array}{r}
\left(\begin{array}{c}
t-1 \\
0
\end{array}\right)\left(\begin{array}{c}
2 m-t \\
m-1
\end{array}\right)+\left(\begin{array}{c}
t-1 \\
1
\end{array}\right)\left(\begin{array}{c}
2 m-t \\
m-3
\end{array}\right)+\cdots+\left(\begin{array}{l}
t-1 \\
t-2
\end{array}\right)\left(\begin{array}{c}
2 m-t \\
m-2 t+3
\end{array}\right) \\
+\left(\begin{array}{l}
t-1 \\
t-1
\end{array}\right)\left(\begin{array}{c}
2 m-t \\
m-2 t+1
\end{array}\right) .
\end{array}
$$

Proof. By applying Lemma 4.2, for each lower-class $\underline{a}$, we group $\underline{a} \cup\{\max \underline{a}+$ $1, \min \underline{a}-1\}$ together. Then the cardinalities of $\left\{\alpha \in \mathcal{O}\left[m_{1 k_{1}} m_{2 k_{2}} \cdots k_{t-1} m_{t}\right]\right.$ : $K_{\alpha}$ is trivial $\}$ and $\{\beta:\{1, \ldots, m-t+1\} \rightarrow\{1, \ldots, m\}: \beta$ is order-preserving $\}$ are the same. Then (i) is proved.

To show (ii), given a $(m+t)$-tuple $\left(x_{1}, x_{2} \ldots, x_{m+t}\right)$ as an integral solution of the equation

$$
x_{1}+x_{2}+\cdots+x_{m+t}=m-1
$$

that satisfy the following conditions:

1. $x_{i} \geq 0$ for all $i \in\{1, \ldots, m+t\}$,

2. for $j \in\left\{m_{1}+1, m_{1}+m_{2}+2, \ldots, m_{1}+\cdots+m_{t-1}+t-1\right\}$, either $x_{j}=x_{j+1}=0$ or $x_{j}, x_{j+1} \geq 1$.

By the condition 2, it guarantees that there is 1-1 corresponding between $\left(x_{1}, x_{2} \ldots, x_{m+t}\right)$ and a map in $\left\{\alpha \in \mathcal{O}\left[m_{11} m_{21} \cdots_{1} m_{t}\right]: K_{\alpha}\right.$ is trivial $\}$. It is clear that there are $\left(\begin{array}{c}2 m-t \\ m-1\end{array}\right)$ solutions for the case $x_{j}=0$ for all $j \in$ $\left\{m_{1}+1, m_{1}+m_{2}+2, \ldots, m_{1}+\cdots+m_{t-1}+t-1\right\}$. For other cases, it can be proved directly. Then our proof is finished. 
For $\alpha \in \mathcal{O}[n]$, we denote $\operatorname{Fix}(\alpha)=\{x \in[n]: x \alpha=x\}$. For $Y \subseteq[n]$, we define

$$
\mathcal{O}_{Y}[n]=\{\alpha \in \mathcal{O}[n]: \operatorname{Fix}(\alpha)=Y\} \text { and } \mathcal{C}_{Y}[n]=\{\alpha \in \mathcal{C}[n]: \operatorname{Fix}(\alpha)=Y\}
$$

The following results were proved by G. Ayik, H. Ayik and M. Koc (see in [1]):

For $Y=\left\{m_{1}, m_{2}, \ldots, m_{r}\right\}$ with $m_{1}<m_{2}<\ldots<m_{r}$, we have

- $\left|\mathcal{O}_{Y}[n]\right|=\prod_{j=1}^{r+1} C_{k_{j}}$, where $k_{1}=m_{1}-1, k_{j}=m_{j}-m_{j-1}(2 \leq j \leq r)$ and $k_{r+1}=n-m_{r}$.

- $\left|\mathcal{C}_{Y}[n]\right|=\prod_{j=1}^{r} C_{k_{j}-1},\left(m_{1}=1\right)$, where $k_{j}=m_{j+1}-m_{j}(1 \leq j \leq r-1)$ and $k_{r}=n-m_{r}+1$.

Lemma 4.4. For $\alpha \in \mathcal{C}\left[m_{k} n\right], K_{\alpha}$ is trivial if and only if one of the following statements hold:

(i) $|(m+\{0,1, \ldots, k, k+1\}) \alpha|=1$.

(ii) $k=1$ and $((m+1) \alpha) \alpha^{-1}=\{m+1\}$.

(iii) $(m) \alpha=m$.

By Lemma 4.4 (iii), it is clear that the set of all maps $\alpha \in \mathcal{C}\left[m_{1 k_{1}} m_{2 k_{2}} \cdots_{k_{t-1}}\right.$ $\left.m_{t}\right]$ such that $\left\{m_{1}, m_{1}+k_{1}+m_{2}, \ldots, m_{1}+k_{1}+m_{2}+\cdots+k_{t-2}+m_{t-1}\right\} \subseteq \operatorname{Fix}(\alpha)$, is $\mathcal{K}$-trivial. By applying the result in [1], the following proposition is obtained.

Proposition 4.5. For $Y=\left\{1, m_{1}, m_{1}+k_{1}+m_{2}, \ldots, m_{1}+k_{1}+m_{2}+\cdots+\right.$ $\left.k_{t-2}+m_{t-1}\right\}, \mathcal{C}_{Y}\left[m_{1 k_{1}} m_{2 k_{2}} \cdots_{k_{t-1}} m_{t}\right]$ is $\mathcal{K}$-trivial with $C_{m_{1}-2} C_{m_{2}-1} \cdots C_{m_{t-1}-1}$ $C_{m_{t}}$ as its cardinality.

Proposition 4.6. Let $\gamma$ be the right identity in $V_{t-1}\left[m_{1 \triangleright} m_{2 \triangleright} \cdots \triangleright m_{t}\right]$. Then $\left\{\gamma \alpha: \alpha \in \mathcal{C}\left[m_{1 k_{1}} m_{2 k_{2}} \cdots_{k_{t-1}} m_{t}\right]\right\}$ is $\mathcal{K}$-trivial with $C_{m_{1}+\cdots+m_{t}-t+1}$ as its cardinality.

Proof. It is clear that for $\alpha \in \mathcal{C}\left[m_{1 k_{1}} m_{2 k_{2}} \cdots_{k_{t-1}} m_{t}\right], \gamma \alpha$ satisfies (i) in Lemma 4.4. Clearly, $\operatorname{ran}(\gamma \beta) \subseteq \operatorname{ran} \gamma$ and $|\operatorname{ran} \gamma|=m_{1}+\cdots+m_{t}-t+1$. That is, $\left\{\gamma \alpha: \alpha \in \mathcal{C}\left[m_{1 k_{1}} m_{2 k_{2}} \cdots_{k_{t-1}} m_{t}\right]\right\}$ is isomorphic to $\mathcal{C}\left[m_{1}+\cdots+m_{t}-t+1\right]$. 


\section{References}

[1] G. Ayik, H. Ayik, M. Koc, Combinatorial results for order-preserving and order-decreasing transformations, Turk J. Math., 35 (2011), 617-625, doi: 10.3906/mat-1010-432.

[2] P.M. Higgins, Combinatorial results for semigroups of order-preserving mappings, Math. Proc. Camb. Phil. Soc., 113 (1993), 281-296.

[3] C.J. Hillar, T. Windfeldt, Fibonacci identities and graph colorings, The Fibonacci Quarterly, 46/47 (2008/2009), 220-224.

[4] J.M. Howie, Products of idempotents in certain semigroups of orderpreserving transformations, Proc. Edinburgh Math. Soc., 17 (1971), 223236.

[5] P. Jitjanakrn, Subsemigroups of order-preserving full transformation: Isomorphism Theorem, Submitted.

[6] A. Laradji, A. Umar, On certain finite semigroups of order-preserving transformation I, Semigroup Forum, 69 (2004), 184-200.

[7] A. Laradji, A. Umar, Combinatorial results for semigroups of orderpreserving full transformations, Semigroup Forum, 72 (2006), 51-62, doi: 10.1007/s00233-005-0553-6.

[8] J.E. Pin, Varieties of Formal Languages, North Oxford Academic, London (1986).

[9] A. Plaza, S. Falcón, Combinatorial proofs of Honsberger-type identities, Int. J. Math Ed. Sci. Tech., 39, No. 6 (2008), 785-792, doi: 10.1080/00207390801986916.

[10] J. Sanwong, The regular part of a semigroup of transformations with restricted range, Semigroup Forum, 83 (2011), 134-146, doi: 10.1007/s00233011-9320-z.

[11] J.S.V. Symons, Some results concerning a transformation semigroup, J. Austral. Math. Soc., 19, Series A (1975), 413-425.

[12] P. Udomkavanich, P. Jitjankarn, A classification of regressive transformation semigroups on chains, Semigroup Forum, 85 (2012), 559-570, doi: $10.1007 / \mathrm{s} 00233-012-9438-7$. 